\title{
EL CEMENTERIO NEOCLÁSICO DEL VI CONDE DE FERNÁN NÚÑEZ: UN EJEMPLO DE DISTINCIÓN SOCIAL Y APROPIACIÓN DEL ESPACIO FUNERARIO*
}

\author{
THE SIXTH COUNT OF FERNÁN NÚÑEZ NEOCLASSI- \\ CAL CEMETERY: A CASE OF SOCIAL DISTINCTION AND \\ APPROPRIATION OF THE FUNERARY SPACE
}

\author{
José Antonio Vigara Zafra \\ UNED. España \\ javigara@geo.uned.es
}

\begin{abstract}
Este trabajo pretende un análisis del cambio en las prácticas funerarias desarrolladas por el VI conde de Fernán Núñez en su villa tras el restablecimiento de los cementerios fuera de poblado. Para ello se estudian las etapas constructivas, las funciones simbólicas y los litigios sociales originados en torno a este novedoso cementerio. Con ello se esperan extraer consecuencias relativas al análisis de las nuevas estrategias de distinción social generadas por las élites nobiliarias durante la Ilustración.

Palabras clave: Nobleza, Distinción social, Prácticas funerarias, Cementerios.
\end{abstract}

This paper tries to analyze the change in burial practices carried out by the sixth Count of Fernán Núñez in his villa after the out-of-town cemeteries reestablishment. To do this, the phases of completion, the symbolic functions and the social disputes resulting from the construction of this new cemetery are described and examined. We claim that this analysis has relevant consequences for the study of the new policies of social distinction of the nobility during the Enlightenment.

Keywords: Nobility, Social distinction, Burial practices, Cemeteries

* Este estudio se ha realizado en el marco del proyecto del Ministerio de Economía y Competitividad: Politicas en tránsito para la legitimación nobiliaria: narrativas de memoria y estética en la gestión del patrimonio artístico de la nobleza española, HAR2012-36751. Agradezco al profesor Antonio Urquízar Herrera sus consejos para mejorar este artículo. 
La Real Cédula de Carlos III de 3 de abril de 1787 proporcionó carta de naturaleza a la obligada implantación de los cementerios fuera de poblado en España, iniciándose, por un lado, un proceso que aspiraba a erradicar los problemas de salubridad pública provocados por las emanaciones nocivas procedentes de los cadáveres que saturaban las iglesias parroquiales y sus espacios aledaños. Por otro, provocó una transformación de las prácticas funerarias heredadas desde la Edad Media, que progresivamente fue restando poder a la iglesia en este ámbito en beneficio del Estado, medidas que en gran parte de Europa ya venían aplicándose desde la segunda mitad del siglo XVIII ${ }^{1}$.

Como es sabido, el detonante de la orden carlotercista estuvo directamente relacionado con el suceso acaecido en la villa guipuzcoana de Pasajes en marzo de 1781, donde se propagó una grave epidemia a consecuencia del elevado número de cadáveres sepultados en la iglesia parroquial. Por este motivo, Carlos III encargó un memorial a la Real Academia de la Historia con el fin de resolver este problema, fructificando en 1786 en el conocido Informe sobre la disciplina eclesiástica antigua y moderna relativa al lugar de sepulturas que junto a la construcción y el reglamento del cementerio del Real Sitio de San Ildefonso en 1785, constituyeron las referencias obligadas para los camposantos edificados con posterioridad.

Con el presente estudio y a través del uso de documentación inédita, pretendemos analizar el cementerio extramuros ideado por Carlos José Gutiérrez de los Ríos y Rohan-Chabot, VI conde de Fernán Núñez, para su villa solariega².

${ }^{1}$ La bibliografía sobre este tema a nivel europeo es abundante. Véase, entre otros, McMANNERS, John: Death and the Enlightenment: changing attitudes to death among Christians and unbelievers in Eighteenth-century France, Oxford, 1981; ARIÉS, Philippe: El hombre ante la muerte, Madrid, 1983, pp. 398-431; ETLIN, Richard: The Architecture of Death: The transformation of the Cemetery in Eighteenth-century Paris, Massachusetts, 1984, pp. 41-100; JENNER, Mark: "Death, Descomposition and Dechristianisation? Public Health and Church Burial in Eighteenth-century England", English Historical Review, vol. CXX, n ${ }^{\circ} .487,2005$, pp. 615-632; ASTON, Nigel: Art and religion in Eighteenth-century Europe, London, 2009, pp. 214-233.

2 Para un análisis biográfico más completo, véase: PÉREZ DE GUZMÁN, Juan: Embajada del Conde de Fernán Núñez en París a los comienzos de la Revolución Francesa, Madrid, 1910; MOUSSET, Albert, Un témoin ignoré de la révolution: le comte de Fernan Nuñez, ambassadeur d'Espagne à Paris (1787-1791), París, 1924; GUTIÉRREZ, Jesús: "Un arte de vivir en el siglo XVIII. La carta del sexto Conde de Fernán Núñez", Dieciocho, 6, 1983, pp. 42-60; MOREL-FATIO, Alfred, PAZ MELIÁ, Antonio: "Biografía del conde de Fernán Núñez", en GUTIÉRREZ DE LOS RÍOS, Carlos José: Vida de Carlos III, Madrid, 1988, vol. II, pp. 331-409; CEPEDA ADÁN, José: "Dos testigos españoles de la Revolución Francesa. El Conde de Fernán Núñez y Pablo de Olavide", en DIEGO GARCÍA, Emilio, GUTIÉRREZ ÁLVAREZ, José, CONTRERAS MIGUEL, Remedios, BULLÓN DE MENDOZA, Alfonso (coords.): Repercusiones de la Revolución Francesa en 
Así, por un lado, centraremos nuestra atención en analizar el proceso constructivo de esta novedosa tipología arquitectónica propia de la Ilustración, destacando su excepcionalidad por ser uno de los primeros ejemplos de cementerio edificado gracias a la iniciativa personal de un noble en la Andalucía de fines del siglo XVIII ${ }^{3}$. Por otro, destacaremos la función simbólica otorgada por el VI conde a este camposanto, que lo concibió como un espacio para la sociabilidad religiosa, destinado a guardar y exhibir la piedad del linaje, imponiendo un nuevo ritual funerario a sus vasallos que aspiraba a desligar el poder de la iglesia en el ámbito funerario en pos de un fortalecimiento señorial en tales prácticas, ejerciendo así sus derechos de patronato frente al concejo municipal de la villa. Asimismo, ofreceremos un nuevo perfil de la figura del VI conde, implicado de forma directa en calidad de arquitecto y mecenas en la configuración y publicitación del cementerio.

Por último, el cambio de centuria llevó aparejado un acentuado absentismo por parte de los titulares de la Casa con respecto a la villa de origen del linaje. Sin embargo, la intención del ayuntamiento de construir un nuevo cementerio a partir de 1858, nos permite analizar las nuevas perspectivas familiares en materia funeraria que tuvo Manuel Falcó d’Adda Valcárcel, III duque de Fernán Núñez, y el conflicto social suscitado con las autoridades municipales consecuencia de sus deseos de exhibición de la pompa nobiliaria.

\section{EL PROYECTO DE CEMENTERIO ILUSTRADO DEL VI CONDE DE FERNÁN NÚÑEZ Y LA EXHIBICIÓN DE LA PIEDAD FAMILIAR}

El VI conde de Fernán Núñez fue un ejemplo paradigmático de ilustrado. Desde su juventud tuvo una vida itinerante que le llevó a visitar gran parte de España y Europa, mostrando un marcado interés por el arte, la cultura y la ciencia. Los puestos diplomáticos que ocupó durante el último cuarto del siglo XVIII,

España, Madrid, 1990, pp. 623-644; PRIETO, Rosario: La Revolución Francesa vista por el embajador de España, Conde de Fernán Núñez, Madrid, 1997; URQUÍZAR HERRERA, Antonio, VIGARA ZAFRA, José Antonio: "La nobleza española y Francia en el cambio de sistema artístico, 1700-1850”, en SAZATORNIL RUIZ, Luis, JIMÉNO, Frédéric: El arte español entre Roma y París (siglos XVIII y XIX). Intercambios artísticos y circulación de modelos, Madrid, 2014, pp. 258-260.

3 RODRÍGUEZ BARBERÁN, Javier: Cementerios de Andalucía: Arquitectura y Urbanismo, Sevilla, 1993, pp. 33-44; GÓMEZ NAVARRO, Soledad: "La construcción de cementerios en la provincia de Córdoba, 1787-1833" en Una Arquitectura para la Muerte. Actas I encuentro internacional sobre los cementerios contemporáneos, Sevilla, 1993, pp. 399-405; GÓMEZ NAVARRO, Soledad: "La construcción de cementerios extramuros en la España Moderna: entre la necesidad y la realidad", en REY CASTELAO, Ofelia; LÓPEZ, Roberto (eds.): El mundo urbano en el siglo de la Ilustración, Santiago de Compostela, 2009, t. II, pp. 163-175. 
primero como embajador en Lisboa y luego en París, afianzaron su gusto por el conocimiento artístico y el mundo académico, plasmándolo en los múltiples proyectos que impulsó, dentro de los cuáles sobresalieron las reformas arquitectónicas destinadas a la mejora de su villa solariega.

La iniciativa del VI conde de construir un cementerio público en Fernán Núñez, se inscribió dentro de la política reformadora que pretendía aplicar a su villa con el objetivo de mejorar sustancialmente el modo de vida de sus vasallos, así como afianzar su vínculo personal con el solar primigenio del linaje. Todo ello gracias a las rentas que heredó en octubre de 1782 tras la muerte de su hermana, Escolástica Gutiérrez de los Ríos y Rohan Chabot, duquesa viuda de Béjar, recibiendo más de cinco millones de reales de vellón ${ }^{4}$.

Sin embargo, la idea primigenia de este cementerio, como en muchos otros municipios, partió de la iniciativa del ayuntamiento de la villa que en diciembre de 1785 informaron al VI conde sobre el estado de ruina y saturación del camposanto parroquial ${ }^{5}$. En enero de 1786, ante la falta de iniciativa de este, trasladaron al conde de Floridablanca, Secretario de Estado, una petición formal de edificación de cementerio en las inmediaciones de la ermita de San Sebastián, a las afueras de la villa ${ }^{6}$, llegando a adjuntar una minuciosa tasación de este proyecto a cargo de José Díaz de Acevedo ${ }^{7}$, a la sazón maestro de obras del VI conde ${ }^{8}$. Pero tras la aceptación del mismo por parte del cabildo catedralicio de Córdoba y ante la falta de liquidez de las arcas municipales ${ }^{9}$, el VI conde comenzó a interesarse por esta iniciativa, así como por el control efectivo del ritual funerario y la asistencia sanitaria de su villa, al constituir estos un adecuado marco para la exhibición del poder familiar ${ }^{10}$.

${ }^{4}$ AHN-SN (Archivo Histórico Nacional, Sección Nobleza, Toledo), Fernán Núñez, C. 2347 , D. 1-11.

${ }^{5}$ AHN-SN, Fernán Núñez, C. 470, D. 10-2.

${ }^{6}$ AHN-SN, Fernán Núñez, C. 470, D. 10-3.

${ }^{7}$ Sobre la figura de José Díaz de Acevedo apenas tenemos datos biográficos y profesionales acerca de su etapa anterior al cargo de maestro obras del VI conde de Fernán Núñez. Tan solo tenemos constancia de que inició su carrera durante el tercer cuarto del siglo XVIII en su ciudad natal, Écija, interviniendo en la fábrica de la iglesia de Santiago, de la iglesia de Santa Bárbara o en la Casa de las Comedias, véase: MORALES, Alfredo José, SANZ, María Jesús, SERRERA, Juan Miguel, VALDIVIESO, Enrique: Guía artística de Sevilla y su provincia, Sevilla, 1981, p. 406; GARCÍA LEÓN, Gerardo: "Planos de Ignacio de Tomás para la iglesia de Santa Bárbara de Écija”, Laboratorio de Arte, 3, 1990, p. 176; FERNÁNDEZ MARTÍN, María Mercedes: "Fiestas en Écija por la proclamación de Carlos IV", Laboratorio de Arte, 11, 1998, p. 599.

8 AHN-SN, Fernán Núñez, C. 470, D. 10-4.

9 AHN-SN, Fernán Núñez, C. 470, D. 10-1.

${ }^{10}$ El VI conde de Fernán Núñez expresó sus intenciones sobre este asunto al vicario de la villa, Andrés Sánchez de Torés, para que lo trasladara a los miembros del 
En efecto, la debilidad económica del concejo propició que se aceptaran cada una de las imposiciones efectuadas por el VI conde, aumentando así su poder efectivo sobre la villa ${ }^{11}$. El primer paso para tener el control de los rituales funerarios de sus vasallos fue apropiarse del patronato de la hermandad de las Ánimas que desde siglos atrás regían vecinos de Fernán Núñez. Para ello, contando con el beneplácito del entonces hermano mayor de la misma, Esteban de Aguado ${ }^{12}$, refundó esta obra pía y los estatutos de la misma, bajo la nueva denominación de hermandad de la Caridad, juntando en esta fundación tanto la reforma del hospital de la Caridad como la construcción del futuro cementerio consagrado a San Sebastián. Lo interesante de este nuevo reglamento radicó en la concentración de poder absoluto en materia asistencial y funeraria en manos de los titulares del linaje, excluyendo taxativamente las injerencias de cualquier autoridad eclesiástica o secular ajena a la propia institución ${ }^{13}$. Entre las principales atribuciones de la hermandad estuvo la gestión del hospital y, sobre todo, la coordinación del ritual de enterramiento de los vecinos de Fernán Núñez. El VI conde pretendía de esta forma diseñar un nuevo modelo de ritual funerario que permitiera visibilizar la piedad de su linaje, habilitando un cortejo que iba desde las casas de los difuntos hasta la iglesia parroquial de Santa Marina, sede del poder religioso de los Fernán Núñez, donde se ofrecería la misa por el muerto con las correspondientes alabanzas a la piedad señorial, siendo trasladado al anochecer al cementerio por nueve hermanos que portarían cuatro faroles pequeños de rosario. Mientras que a los pobres a quienes solo se diga este ultimo responso y los que quisiesen se les haga el oficio en la Hermita del mismo cimenterio, y se les llevara en derechura a él ${ }^{14}$.

ayuntamiento en octubre de 1786, véase: AHN-SN, Fernán Núñez, C. 470, D. 1-4: "Para esto es necesario que por la villa se me represente el estado en que se halla y la necesidad de remediarlo por medio de un nuevo establecimiento util, en los terminos que yo lo crea mas conveniente à cuio fin con permiso del Señor Obispo, ceden desde luego a mi Casa el Patronato para siempre, á fin de que arreglandolo yo todo del modo que crea mas conveniente, haga de un respetable esqueleto expuesto a su ruina, una fundacion util a aquellos vecinos, á que estan todos prontos a contribuir con sus limosnas, y asistencia personal, siempre que se erigiere nueva Hermandad para alivio de los pobres. Mi proiecto es verificar la obra del cimenterio de Sn. Sebastian, y reuniendole a la Caridad [hospital], establecer una Hermandad util de caridad de que el Señor de la Casa seria el Hermano mayor nato, distinguiendolo de modo que dandose la mano estos dos caritativos establecimientos, se cuidasen a prosperar recíprocamente".

11 AHN-SN, Fernán Núñez, C. 470, D. 10-5.

12 AHN-SN, Fernán Núñez, C. 470, D. 17-2 y 6.

13 AHN-SN, Fernán Núñez, C. 470, D. 8.

${ }^{14}$ El ritual establecido por el VI conde de Fernán Núñez imita el aprobado el 9 de febrero de 1785 para el cementerio del Real Sitio de San Ildefonso, véase: AHN-SN, Fernán Núñez, C. 470, D. 17-3. Asimismo, el VI conde de Fernán Núñez, con el objetivo de dar ejemplo y promover entre sus vasallos las ventajas de este tipo de camposanto extramuros 
El VI conde, tras establecer esta fundación, inició desde su cargo de embajador en Lisboa la configuración del cementerio. En ese sentido, la historiografía, partiendo de la errónea descripción efectuada por Pascual Madoz en su Diccionario geográfico-estadístico-histórico de España ${ }^{15}$, ha señalado que si bien el mecenazgo de esta edificación estuvo a cargo del conde, la autoría del mismo es anónima, indicando la más que posible participación en su traza de algún arquitecto del entorno de la academia, consecuencia del carácter clasicista que presentó esta obra ${ }^{16}$. Solamente, en fechas recientes, Carlos Saguar Quer ha apuntado la posibilidad de que tras el diseño de este proyecto estuviera la figura del VI conde de Fernán Núñez ${ }^{17}$. Efectivamente, gracias a la documentación inédita manejada para este estudio hemos podido confirmar la intuición de este autor, constituyendo Carlos José Gutiérrez de los Ríos tanto el mecenas como el arquitecto del cementerio ideado para su villa.

Entre los aristócratas de su tiempo, el VI conde fue una figura singular no solo por su exhaustivo conocimiento teórico de los nuevos planteamientos arquitectónicos y urbanísticos que se estaban generando tanto en Europa como en España ${ }^{18}$, también por las destrezas prácticas exhibidas a la hora de construir

de la villa, dictaminó que los componentes de la hermandad debían acudir al cementerio todos los viernes, domingos y días festivos, encabezando un vía crucis en el claustro para los que gusten á rezar los pasos, ó orar por la animas de sus difuntos, y por los que por ellos pidan, se alcanzarán de los prelados eclesiásticos las indulgencias correspondientes, véase: AHN-SN, Fernán Núñez, C. 470, D. 5.

${ }^{15}$ MADOZ, Pascual: Diccionario geográfico-estadístico-histórico de Andalucía. Córdoba, Valladolid, 1987, p. 160.

${ }^{16}$ SAGUAR QUER, Carlos: "Carlos III y el restablecimiento de los cementerios fuera del poblado", Fragmentos, no 12, 13 y 14, 1988, p. 254; SAGUAR QUER, Carlos: Arquitectura funeraria madrileña del siglo XIX, Madrid, 1989, tesis doctoral, pp. 20-21; SAGUAR QUER, Carlos: "La aparición de una nueva tipología arquitectónica: el Cementerio", en El arte en tiempo de Carlos III, Madrid, 1989, pp. 211-213; BERMEJO LORENZO, Carmen: Arte y arquitectura funeraria. Los cementerios de Asturias, Cantabria y Vizcaya (1787-1936), Oviedo, 1998, p. 48; MORENO ATANCE, Ana María: Cementerios murcianos: Arte y arquitectura, Madrid, 2005, tesis doctoral, p. 633.

${ }^{17}$ SAGUAR QUER, Carlos: "La Casa de los Muertos: El Cementerio en la España del siglo XIX”, en LÓPEZ-GUADALUPE MUÑOZ, Juan Jesús (ed.): Memoria de Granada. Estudios en torno al Cementerio, Granada, 2006, pp. 282-283.

${ }_{18}$ A través de los diarios de viaje del VI conde de Fernán Núñez podemos analizar la implantación de los nuevos planteamientos artísticos en la Europa del último cuarto del siglo XVIII, así como las relaciones establecidas por el conde con agentes vinculados a las artes de la talla de Antonio Ponz, Gaspar de Jovellanos, el V marqués de Montehermoso o el III marqués de Ureña, véase: AHN-SN, Fernán Núñez, C. 2033, D. 1 al 13. El propio Ponz al ensalzar la modernidad arquitectónica del palacio del VI conde de Fernán Núñez, la atribuyó a los conocimientos que dicho Señor ha adquirido en sus largos viages por Europa, 
el palacio familiar ${ }^{19}$. No en vano, desde 1770 tuvo participación directa en los debates generados en torno al nuevo estilo clasicista en el seno de la Real Academia de Bellas Artes de San Fernando en calidad de consiliario ${ }^{20}$.

Sin embargo, como hemos señalado, desarrolló gran parte de su vida alejado de sus estados. Por este motivo, aunque trazó cada uno de los proyectos destinados a su villa, tuvo que delegar la construcción y finalización de los mismos en personas de su entera confianza. De este modo, la supervisión directa de las obras recayó sobre el administrador de la villa, Joaquín de Luna ${ }^{21}$, mientras que la dirección de la fábrica de los edificios estuvo a cargo de José Díaz de Acevedo, que desde la década de los setenta trabajó para el conde en obras de distinta índole ${ }^{22}$.

En el primer diseño del cementerio formalizado en noviembre de 1786 intervinieron los agentes citados anteriormente ${ }^{23}$. Así, el conde diseñó el plano del cementerio que remitió a Joaquín de Luna, y este lo entregó a su vez a José Díaz de Acevedo para que, tras el pertinente reconocimiento de los terrenos, efectuara el presupuesto del mismo. Este primer proyecto de cementerio se adaptó a los principios que posteriormente se divulgaron en la Real Cédula de 3 de abril de 1787 , proyectándolo en un espacio con buena ventilación a las afuera de la villa y aprovechando como capilla la antigua ermita de San Sebastián ${ }^{24}$. José Díaz de Acevedo tasó las obras del mismo en 168.343 reales de vellón, tratándose pues de un edificio de planta rectangular cuya entrada estaría localizada en la zona de la ermita, siendo esta reformada desde los cimientos y agregando una serie de dependencias anexas que servirían de vivienda para el vigilante del edificio. Tras la capilla se localizaría un amplio recinto con claustro alrededor para los enterramiento comunes, cerrado este con una verja de hierro con cuatro accesos al mismo, que coincidirían con las cuatro calles empedradas y flanqueadas de cipreses en que estaría dividido el cementerio. Finalmente, en el extremo opuesto del

véase: PONZ, Antonio: Viaje de España, en que se da noticia de las cosas más apreciables, y dignas de saberse, que hay en ella, Madrid, tomo XVII, 1792, p. 152.

19 VIGARA ZAFRA, José Antonio: "El palacio del VI conde de Fernán Núñez: La arquitectura como exaltación simbólica del linaje durante la Ilustración", Tiempos Modernos: revista electrónica de Historia Moderna, vol. 8, nº. 29, 2014, (en prensa).

${ }^{20}$ BÉDAT, Claude: Los Académicos y las Juntas, 1752-1808, Madrid, 1982, pp. 8 y ss.; BÉDAT, Claude: La Real Academia de Bellas Artes de San Fernando (1744-1808). Contribución al estudio de las influencias estilísticas y de la mentalidad artística en la España del siglo XVIII, Madrid, 1989, pp. 371-398.

${ }^{21}$ Sobre el régimen administrativo del estado de Fernán Núñez, el VI conde redactó una serie de directrices concretas para cada uno de sus sirvientes. Para el caso del gobernador, una de sus principales adscripciones fue la del mantenimiento de las propiedades del señor, véase: AHN-SN, Fernán Núñez, C. 470, D. 29.

${ }^{22}$ AHN-SN, Fernán Núñez, C. 470, D. 23.

${ }^{23}$ AHN-SN, Fernán Núñez, C. 470, D. 10-11.

${ }^{24}$ AHN-SN, Fernán Núñez, C. 220, D. 7-11. 
claustro habilitarían una serie de nichos para las personalidades de la villa que pretendieran tener un entierro distinguido ${ }^{25}$.

Sin embargo, el VI conde desestimó este primer proyecto de camposanto como consecuencia de un cambio en las políticas de creación y transmisión de la memoria familiar que pretendía implantar en el ámbito funerario. Así, lejos de seguir con la tradición funeraria de la Casa en la iglesia parroquial de Santa Marina y frente a la oposición del estamento nobiliario a aceptar la nueva tipología de cementerios fuera de poblado, perdiendo así sus derechos de sepultura en las iglesias, optó por modificar el primer diseño de camposanto para su villa, incluyendo en el mismo como gran novedad un panteón familiar à fin de que siendo los primeros á dar el exemplo, no tengan los demás reparo, ni desdeñen de seguirle ${ }^{26}$. De esta forma, evidenció su continuo afán de distinción al constituir el panteón un edificio mucho más suntuoso que la antigua iglesia parroquial.

En enero de 1787, el VI conde finalizó los planos definitivos para el cementerio, cuya morfología evidenció la influencia directa del cementerio de Turín diseñado por Giuseppe Oglianico en $1781^{27}$. El recinto consistió en un amplio espacio rectangular ${ }^{28}$, con una entrada principal con frontón triangular y pórtico tetrástilo de reminiscencias clásicas que daría acceso a la capilla de planta centralizada compuesta de tres altares, sacristía y dependencias para el sacristán. A continuación, se accedería a un gran patio rodeado por una columnata, en cuyo centro se habilitaría una calle de cipreses perpendicular al eje de la capilla que dividiría en dos partes el cementerio destinado a las sepulturas comunes. Tras esto, se ubicarían los espacios distinguidos, constando de catorce sepulturas independientes para personalidades de la villa que quisieran comprarlas y, al fondo, el panteón

${ }^{25}$ AHN-SN, Fernán Núñez, C. 470, D. 10-10.

${ }^{26}$ ESPINALT GARCÍA, Bernardo: El Atlante Español ó Descripción general de todo el Reyno de España. Parte segunda, en que se concluye el Reyno de Córdoba, y empieza el de Jaén, Madrid, 1787, vol. XII, p. 49. De este modo, el VI conde de Fernán Núñez coincidió con los pensamientos más renovadores de ilustrados de la talla de Gaspar Melchor de Jovellanos que renunció al derecho de sepultura en la capilla de los Reyes de la iglesia de San Pedro en Gijón, véase: CEÁN BERMÚDEZ, Juan Agustín: Memorias para la vida del Excmo. Señor D. Gaspar Melchor de Jovellanos y noticias analíticas de sus obras, Madrid, 1814, pp. 236-237.

${ }^{27}$ El XI duque de Villahermosa, Juan Pablo de Aragón-Azlor, a la sazón conocido del VI conde de Fernán Núñez, distribuyó en España el modelo de camposanto turinés gracias al encargo de los planos del mismo a los académicos Dámaso Santos Martínez y Manuel Rodz, véase: Noticias y reflexiones del Excelentísimo Señor Duque de Villahermosa, sobre los cementerios fuera de los poblados, y en especial sobre los establecidos extramuros de Turín, Madrid, 1786.

${ }^{28}$ El cementerio construido por el VI conde de Fernán Núñez tuvo una superficie total de 55 varas castellanas de longitud y 32 de latitud, es decir, 15.840 pies cuadrados, véase: AHN-SN, Fernán Núñez, C. 220, D. 7-12. 
de planta centralizada de los señores de Fernán Núñez con treinta y seis nichos, altar y sacristía propia. Finalmente, en un ángulo de la parte posterior del recinto situaría el osario configurado a modo de patio con un pozo profundo donde depositar los huesos de los cadáveres ${ }^{29}$. Este diseño revela el conocimiento que tuvo el VI conde de los proyectos de arquitectura funeraria generados desde 1784 en el seno de la Real Academia de Bellas Artes de San Fernando para las pruebas y concursos de arquitectura ${ }^{30}$. De hecho, como en la mayoría de estos, el configurado para la villa de Fernán Núñez muestra con claridad las reminiscencias a la antigüedad clásica, especialmente la influencia directa del Panteón de Roma, tendencia esta que fue común en el resto de Europa ${ }^{31}$.

Sin embargo, lo excepcional de este proyecto fue que, frente a los diseñados en la academia fernandina, logró pasar de la utopía del papel a la realidad de la piedra. Las obras comenzaron en abril de 1787, y en junio el conde visitó la villa de Fernán Núñez para preparar su desplazamiento a París en calidad de embajador. De esa forma, pudo valorar en primera persona el progreso de la fábrica del cementerio, fundamentalmente ceñida a la construcción de los muros perimetrales y el claustro del mismo, pero sin modificar la ermita de San Sebastián ya que hasta lo ultimo no se ha de hacer por no hechar á tierra la vieja afin que en el interior no se deje de decir Misa en ella ${ }^{32}$. Asimismo, tuvo una especial preocupación por la utilización de los espacios arquitectónicos como escenografías para proyectar el poder individual y familiar, entendiendo el cementerio como un espacio idóneo para exhibir la distinción social de la Casa. Por ello, centró sus instrucciones en la paulatina finalización del recinto y, especialmente, en la configuración de los elementos decorativos de los espacios más representativos ${ }^{33}$.

${ }^{29}$ AHN-SN, Fernán Núñez, C. 470, D. 10-13.

${ }^{30}$ GONZÁLEZ DÍAZ, Alicia: "El cementerio español en los siglos XVIII y XIX", Archivo Español de Arte, t. 43, nº 171, 1970, pp. 289-320; SAGUAR QUER, Carlos: "Ciudades de la memoria. Proyectos de arquitectura funeraria de la Real Academia de Bellas Artes de San Fernando", Boletín de la Real Academia de Bellas Artes de San Fernando, $\mathrm{n}^{\circ} .81,1995$, pp. 450-476.

${ }^{31}$ SAMBRICIO, Carlos, La arquitectura española de la Ilustración, Madrid, 1986, pp. $1-32$.

32 AHN-SN, Fernán Núñez, C. 220, D. 7-11.

${ }_{33}$ AHN-SN, Fernán Núñez, C. 470, D. 11-1: “25․ En el cementerio se hara ahora la cerca exterior toda completa: se suviran hasta el raso los cimientos del Panteon de los SSres. y quartos anexos. Se harán dos sepulturas en el Claustro. Se levantará el parapeto de todo el, y pondrà la berja de madera. Se empedrara el claustro dejandolo por ahora al descubierto. Luego se seguira el Panteon hasta acavarle. Despues se cubriran los claustros, y al fin se haran la capilla y quarto anexos hasta su conclusion, à 12.000 reales de gasto al año nada mas en este ramo, despues de hecho este primer gasto.

$26^{\circ}$. En el frente interior del Panteon, frente de la puerta se pondra una lapida grande de marmol negro sobre un pedestal todo de relieve de quatro dedos sobre la pared en que 
En 3 de junio de 1787, se concluyeron los paramentos exteriores del cementerio, faltando por finalizar tan solo el panteón familiar y el pórtico de la fachada principal $^{34}$. Por último, sabemos que la fábrica del recinto continuó hasta finales de 1790 , fecha en la que el gasto total de lo construido ascendía a 78.465 reales de vellón y, con toda probabilidad, cesaron estas inversiones económicas fruto de los gastos que exigía la embajada del conde en París ${ }^{35}$. Así, a partir de ese momento carecemos de documentación que atestigüe la morfología final de lo edificado, aunque el cementerio debió funcionar con total normalidad para el común de los habitantes de la villa puesto que entre julio de 1787 y diciembre de 1809 se verificaron con regularidad los pagos a los vigilantes ${ }^{36}$. Por tanto, es probable que se hubiera finalizado todo el perímetro exterior del recinto, así como el claustro que circundaba la parte habilitada para las sepulturas comunes. Mientras que el panteón destinado a la familia condal se inició, pero deducimos que no llegaron a finalizarlo ya que hasta 1858 se conservó en las dependencias del palacio familiar la lápida conmemorativa que debía ubicarse en la fachada del citado panteón. Y, además, refuerza esta tesis el hecho de que a la muerte del VI conde en febrero de 1795 no se cumpliera con su voluntad de ser enterrado allí, recibiendo sepultura en la iglesia de San Andrés en Madrid ${ }^{37}$.

diga: A. O. Y. G de Dios, Reynando Carlos $3^{\circ}$. y siendo sumo Pontifice Pio $6^{\circ}$. puso la primera piedra de este cementerio publico y Panteon particular para su familia que erigio a su costa el Excmo. Sor. Dn. Carlos Gutierrez de los Rios, asistiendo a este acto la Excma. Sra. Da. Maria de la Esclavitud, su esposa, sus dos hijos Dn. Carlos y Dn. Joseph, el Governador General del Estado, el Theniente Coronel D. Joaquin de Luna, el clero, y justicia de esta villa el dia 5 de mayo de 1787.

$27^{\circ}$. Sobre la puerta del Panteon se pondran las armas en piedra como las de la fachada de Palacio de bajo en la emposta de la puerta este escrito: Descansan con los suyos. En la fachada de la Hermita se pondrán las armas, y sobre la puerta de ella una calavera con dos huesos debajo en cruz, atributo de la muerte, y en el atrio enfrente de la puerta que va al quarto del sachristan otra puerta figurada que sea una lapida con la misma inscripcion que la que esta por dentro del panteon arriba".

${ }^{34}$ AHN-SN, Fernán Núñez, C. 220, D. 7-11. Sin embargo, un informe fechado el 17 de mayo de 1858 por el administrador del III duque de Fernán Núñez, José de Villafranca, atestigua la conclusión del pórtico de la entrada principal, veáse: AHN-SN, Fernán Núñez, C. 220, D. 7-13: "Finalmente tambien devo decir á V. E. que la fachada de la obra que se principio y es lo que queda en pie de ella, no está conforme con el proyecto, pues los intercolumnios estan unidos por arcos y en el dibujo no aparece así; del mismo modo que en el centro ó campo del fronton donde se significa en el dibujo una calavera, se encuentra colocado el escudo de armas de la Casa, y en estos almacenes se conserva la lapida de jaspe negro con la inscripcion que estaba preparada sin duda para ponerla sobre la puerta".

35 AHN (Archivo Histórico Nacional, Madrid), Estado, Leg. 3995, Lib. IV, D. 853.

${ }^{36}$ AHN-SN, Fernán Núñez, C. 469, D. 7; AHN-SN, Fernán Núñez, C. 220, D. 7-12.

${ }^{37}$ AHPM (Archivo Histórico de Protocolos, Madrid), Pr. 24.836, fols. 477r.v. 
Finalmente, la llegada a la titularidad de su hijo, el VII conde y I duque de Fernán Núñez, Carlos José Gutiérrez de los Ríos y Sarmiento, residente en la corte de Madrid y embajador en Londres y París sucesivamente, supuso el inicio de un proceso de absentismo con respecto a la villa de origen del linaje, abandonando así gran parte de las iniciativas impulsadas por el VI conde. Y el cementerio no fue una excepción, desde la epidemia de 1815 fue cedido a los vecinos de la villa que lo utilizaron sin ningún tipo de $\operatorname{control}^{38}$, incluso en su interior se instaló sin autorización la hermandad del Rosario ${ }^{39}$, hallándose hacia 1818 en un estado de extrema dejadez, tal como informaba el apoderado general del I duque de Fernán Núñez, Francisco Javier de Ochoa ${ }^{40}$.

\section{LA PUBLICITACIÓN DE LAS VIRTUDES PERSONALES A TRAVÉS DE LA ARQUITECTURA: EL CEMENTERIO DE FERNÁN NÚÑEZ EN EL ATLANTE ESPAÑOL}

El camposanto del VI conde no llegó a finalizarse de acuerdo al proyecto definitivo diseñado por él. Sin embargo, eso no fue un obstáculo para que a nivel simbólico constituyera un efectivo ejemplo de la utilización de la arquitectura como un medio para la distinción social. Así, el 5 de mayo de 1787 con motivo de colocar la primera piedra del cementerio se desarrolló una ostentosa ceremonia que partió del palacio familiar. Dicha comitiva estuvo formada por la familia del conde al completo, amigos como el III marqués de Ureña, las principales autoridades civiles y eclesiásticas de la provincia de Córdoba y gran número de los habitantes de la villa, finalizando el acto con la bendición y colocación en los terrenos aledaños a la capilla de San Sebastián de una caja a modo de cápsula del tiempo que contenía la memoria de constitución del cementerio y unas monedas de plata de curso ${ }^{41}$.

Pero el VI conde en su afán por publicitar tan excepcional iniciativa pensó desde un primer instante en traspasar el ámbito local de su villa, publicitando así su piedad y carácter ilustrado entre un público más amplio. A esto se suma que, por esas fechas, tras su periplo diplomático en Lisboa, necesitaba promocionarse para aspirar a cargos de mayor importancia, en concreto deseaba recalar en las embajadas de Londres o París. De este modo, concibió las reformas ilustradas emprendidas en su villa como un instrumento válido para exhibir su imagen social, destacando la edificación del cementerio público como uno de los principales elementos indicativo de su magnificencia y modernidad. Por ello, con el objetivo de divulgar estos proyectos, promovió la publicación de una descripción de

\footnotetext{
38 AHN-SN, Fernán Núñez, C. 220, D. 7-1.

39 AHN-SN, Fernán Núñez, C. 220, D. 7-13.

40 AHN-SN, Fernán Núñez, C. 470, D. 29-3.

${ }^{41}$ AHN-SN, Fernán Núñez, C. 220, D. 7-11.
} 
Fernán Núñez en El Atlante Español de Bernardo de Espinalt García, que resultaría un excelente escaparate para la exhibición de sus virtudes como prohombre ilustrado, donde la palabra y, fundamentalmente, la imagen se aunarían para ofrecer una visión de la villa modelada según su conveniencia personal y política $^{42}$. Resultando especialmente interesante su ahínco por distribuir dicha obra entre los aristócratas parisinos gracias a la intermediación del Ministro de Asuntos Exteriores, el conde de Montmorin, ya que le permitiría darse a conocer entre la nobleza francesa meses antes de asumir el cargo de embajador alli ${ }^{43}$.

La descripción de la villa fue escrita por el vicario de su administración, Juan García y Castro, y supervisada por el propio conde en dos ocasiones antes de ser enviada a Bernardo de Espinalt para su publicación. Pero, tan interesante como el texto fueron las imágenes que lo acompañaron. Se trató de tres vistas de Fernán Nuñez encargadas a su pintor de cámara, Vicente Mariani, que grabó una de ellas, La vista de la villa de Fernán Núñez desde encima del batán antiguo, mientras que las dos restantes alusivas al palacio familiar fueron grabadas en Madrid a finales de 1786 por el académico Juan Fernando Palomino. Y junto a estas, se incluyó también el grabado del proyecto de cementerio ideado por el VI conde ${ }^{44}$. Así, en su conjunto, estas estampas respondieron a una imagen idealizada de la villa, diseñada a instancias del conde, pretendiendo presentar una visión modélica de sus iniciativas aún por terminar, sirviendo de esta forma a sus políticas de ostentación social.

\section{EL CEMENTERIO MUNICIPAL DE FERNÁN NÚÑEZ: CONFLICTIVIDAD SOCIAL EN EL CONTROL DEL ESPACIO FUNERARIO EN LA SEGUNDA MITAD DEL SIGLO XIX}

Como hemos señalado, el cambio de centuria representó la ruina del cementerio iniciado por el VI conde, consecuencia del absentismo de sus sucesores en el título con respecto a la villa seminal del linaje, centrados estos en los asuntos políticos, sociales y artísticos de la corte madrileña, donde formaron parte de las más significativas personalidades de las élites aristocráticas de la capital durante la segunda mitad del siglo XIX ${ }^{45}$.

${ }^{42}$ ESPINALT GARCÍA, Bernardo: El Atlante español..., op. cit., vol. XII, pp. 5-63.

43 AAE (Archives du Ministère des Affaires Etrangères, París), Correspondance politique, Espagne, vol. 624, fol. 196r-197r.

${ }^{44}$ Resulta probable que el grabado del cementerio de Fernán Núñez fuera obra de Vicente Mariani ya que sabemos el interés del VI conde por incentivar el progreso de la técnica del grabado en su pintor de cámara, véase: ARABASF (Archivo Real Academia de Bellas Artes de San Fernando, Madrid), Leg. 1/44/1.

${ }^{45}$ VÁZQUEZ, Oscar: Inventing the Art Collection. Patrons, Market, and the State in Nineteenth-Century Spain, Pennsylvania, 2001, pp. 159-185; PRADO HIGUERA, 
El 11 de abril de 1858, el alcalde de Fernán Núñez, Adolfo Darhan y Gastelú, planteó la necesidad de construir un nuevo cementerio sobre las ruinas del configurado por el VI conde, ya que los enterramientos en esa zona habían continuado, aunque sin orden ni direccion algunas, y mirado dicho sitio con tanta indiferencia que los perros y otros animales se han cebado más de una vez en los cadáveres alli enterrados ${ }^{46}$. De tal manera que encargaron el diseño del mismo al arquitecto provincial de Córdoba, Pedro Nolasco Meléndez ${ }^{47}$, quien incluso efectuó un primer plano del recinto sin comunicarlo al III duque de Fernán Núñez, Manuel Falcó d'Adda Valcárcel, obviando así sus derechos sobre la propiedad de unos terrenos que aún pertenecían a la $\mathrm{Casa}^{48}$. Por este motivo, a través de su administrador, José de Villafranca, inició un pleito para hacer valer sus prerrogativas. Por ello, el consistorio municipal tuvo que transigir y efectuar una petición formal de cesión de los mencionados terrenos, formalizándose el 4 de abril de $1860^{49}$, con la única condición por parte del III duque de reservarse solo un sitio preferente para mi Casa ó Familia ${ }^{50}$.

Sin embargo, las obras estuvieron en todo momento bajo la supervisión directa del consistorio municipal y junto al arquitecto, Pedro Nolasco Meléndez, obviaron la intención del III duque de ubicar el panteón familiar en un espacio preferente del cementerio. Así, el nuevo camposanto debía ser un recinto de planta rectangular, ubicando el panteón de los señores de Fernán Núñez tras la fachada principal y justo detrás la capilla, localizándose al fondo el espacio para las fosas comunes ${ }^{51}$. Pero el arquitecto no cumplió con este primer proyecto inicial y las obras continuaron sin atender las demandas de la Casa ducal. Así, el 21 de abril de 1860, José de Villafranca, ante el avanzado estado que presentaban las

Cristina: El Todo Madrid: La corte, la nobleza y sus espacios de sociabilidad en el siglo XIX, Madrid, 2013.

${ }^{46}$ AHN-SN, Fernán Núñez, C. 220, D. 7-1.

${ }^{47}$ Sobre la figura de Pedro Nolasco Meléndez y la remodelación de Córdoba durante la segunda mitad del siglo XIX, véase: GARCÍA VERDUGO, Francisco: Córdoba, burguesía y urbanismo. Producción y propiedad del suelo urbano: El sector de Gran Capitán, 1859-1936, Córdoba, 1992, pp. 93-137; MARTÍN LÓPEZ, Cristina: Córdoba en el siglo XIX. Modernización de una trama histórica, Córdoba, 1990, pp. 43-234.

${ }^{48}$ LUNA HIDALGO, José Antonio; MIRANDA ÁlVAREZ, Pedro: "Un proyecto de panteón para la casa ducal de Fernán Núñez", en Homenaje a Juan Gómez Crespo, Fernán Núñez, 1985.

49 AHN-SN, Fernán Núñez, C. 220, D. 7-9.

${ }^{50}$ AHN-SN, Fernán Núñez, C. 220, D. 7-16. Entendemos que el III duque de Fernán Núñez tenía pensado desde 1858 habilitar un nuevo panteón para los señores de la Casa, como consecuencia de un incidente suscitado con la autoridad eclesiástica que reclamaba la potestad de las llaves del panteón de la iglesia parroquial de Santa Marina en manos del administrador ducal, véase: AHN-SN, Fernán Núñez, C. 2118, D. 59-19.

${ }^{51}$ AHN-SN, Fernán Núñez, C. 220, D. 7-8. 
obras, comunicó al duque que debían renunciar a la idea primigenia de instalar el panteón familiar en el centro de la construcción debido a sus excesivas dimensiones $^{52}$, quedando tan solo la opción de situarlo en uno de los laterales de la entrada, haciendo pareja con el que se dedicaría a las sepulturas de los sacerdotes ${ }^{53}$. Este hecho evidenció con claridad la pérdida de poder del duque con respecto al consistorio municipal, sentando las bases para el posterior abandono definitivo de la vinculación ducal con la villa en materia funeraria.

Para remediar esta situación, el arquitecto propuso habilitar un panteón subterráneo delante de la capilla del cementerio ${ }^{54}$, lo cuál fue rechazado por no cumplir con las expectativas del III duque. Este, finalmente, en 22 de junio de 1864 planteó la posibilidad de disponer el panteón en la capilla de Santa Escolástica del palacio familiar, pero encontró, de nuevo, la oposición del ayuntamiento que vetó dicha propuesta, amparándose en la prohibición de enterramiento en iglesias, cementerios o panteones ubicados dentro de poblado ${ }^{55}$.

Finalmente, el III duque ante la incapacidad para construir un panteón familiar en Fernán Núñez, fruto de la fuerte oposición municipal, se desvinculó por completo de esta idea, encargando en 1879 al arquitecto Francisco de Cubas y González un ostentoso panteón neogótico en los terrenos familiares de La Alameda en Madrid ${ }^{56}$, que puso fin a las políticas de distinción social de los Fernán Núñez con su villa solariega. Se demostraba así el inevitable proceso de absentismo de este linaje con respecto a sus posesiones señoriales, quedando reducidas a un mero interés económico y, por tanto, desprovistas de la función simbólica que tuvieron durante tantos años.

Fecha de recepción: 15 de septiembre de 2015.

Fecha de aceptación: 20 de noviembre de 2015.

52 AHN-SN, Fernán Núñez, C. 220, D. 7-5.

${ }^{53}$ AHN-SN, Fernán Núñez, C. 220, D. 7-3.

${ }^{54}$ Ibídem.

${ }^{55}$ AHN-SN, Fernán Núñez, C. 220, D. 7-4.

${ }^{56}$ SAGUAR QUER, Carlos: Arquitectura funeraria madrileña..., op. cit., pp. 418-419. 


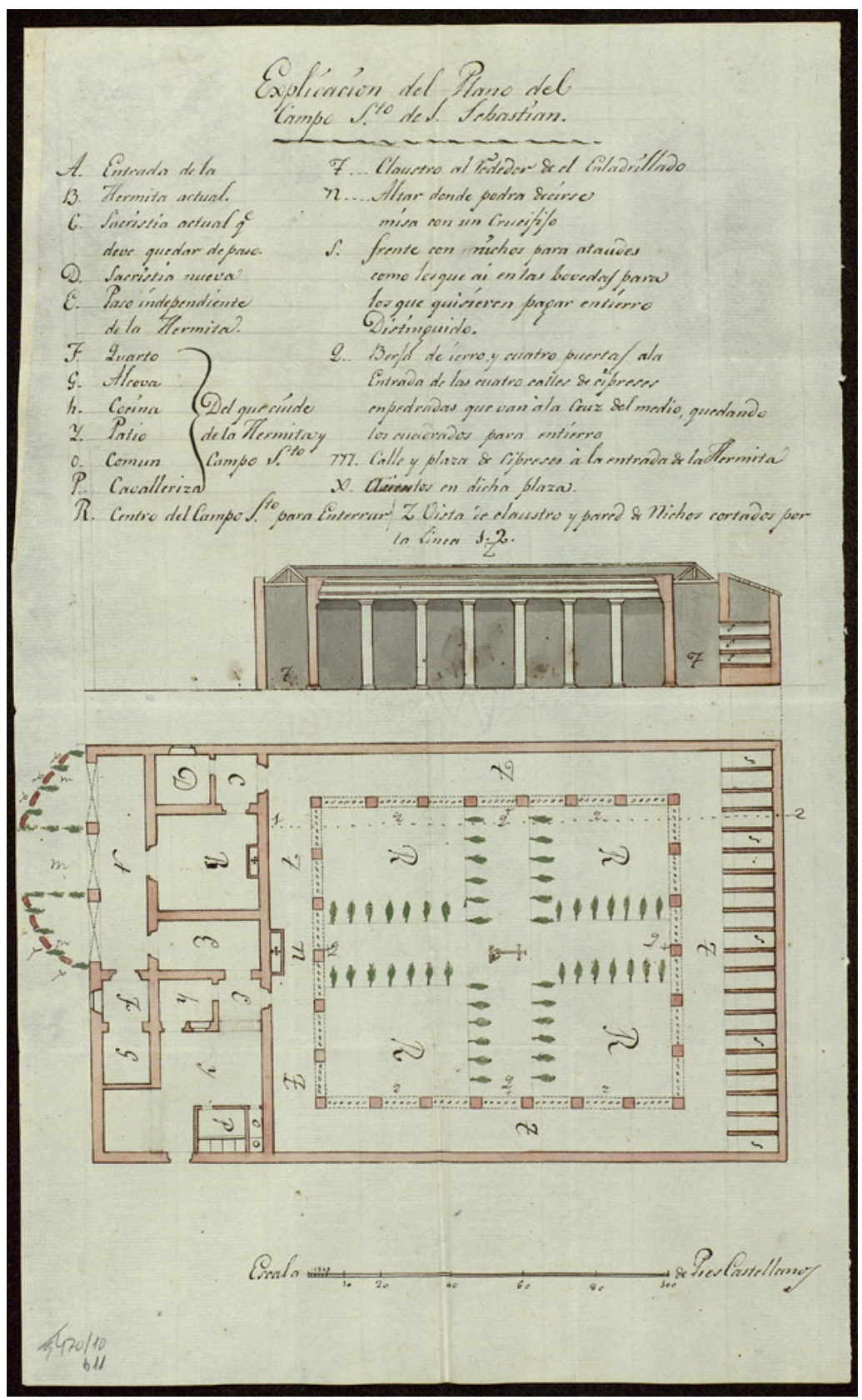

Figura 1. Plano del cementerio de Fernán Núñez, VI conde de Fernán Núñez, 1786, AHN-SN, Toledo. 


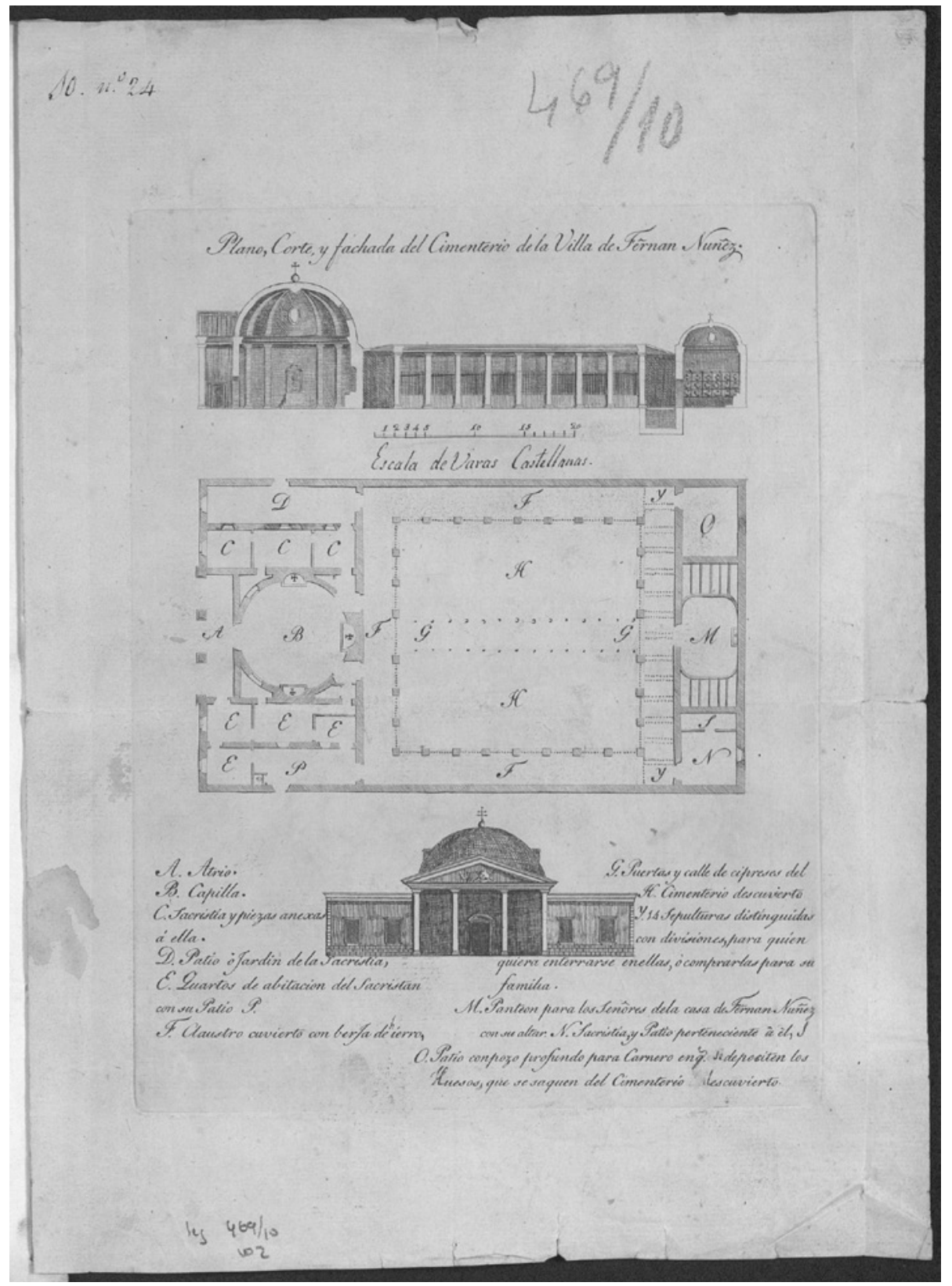

Figura 2. Plano del cementerio de Fernán Núñez, Anónimo, 1787, AHN-SN, Toledo. 


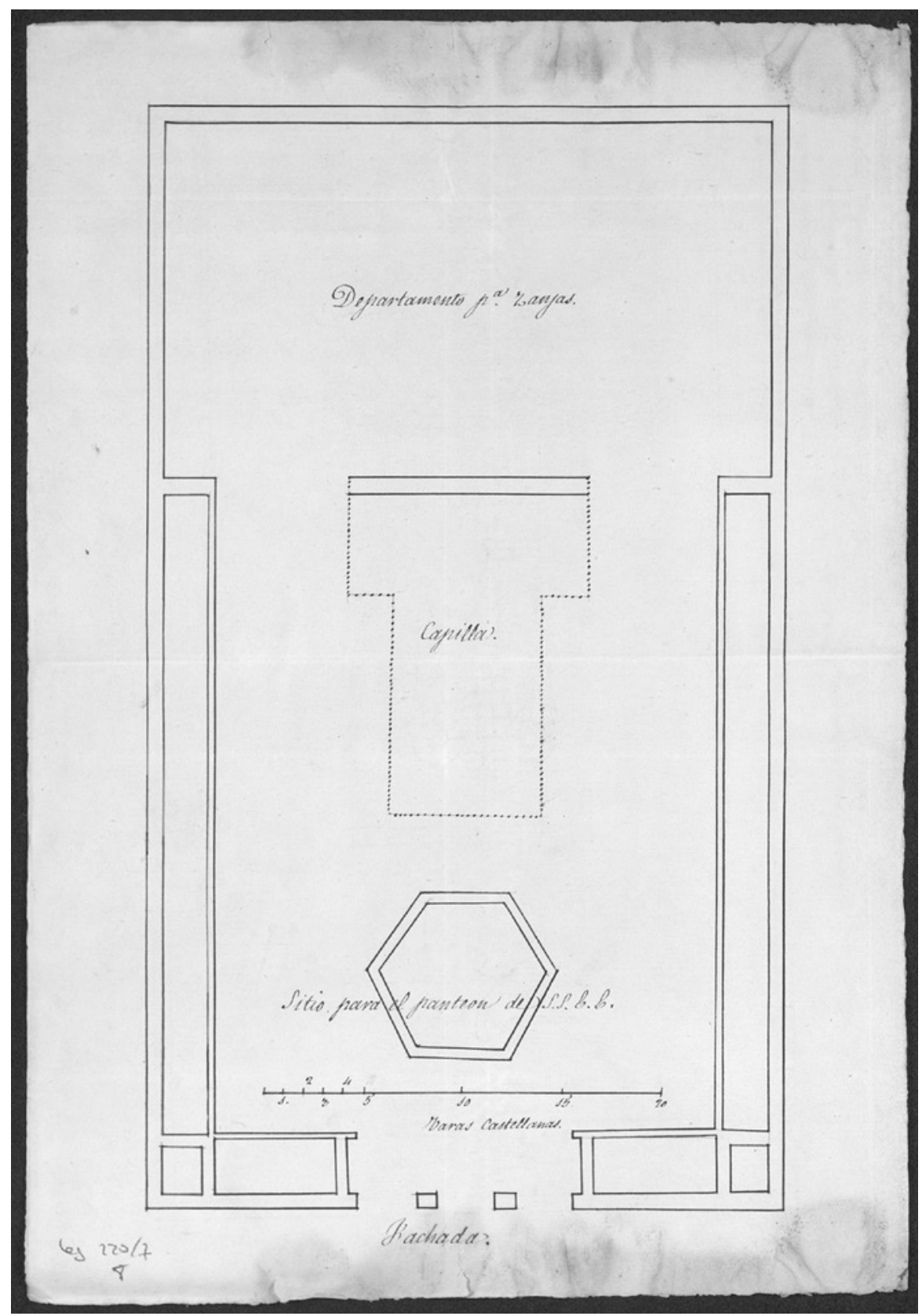

Figura 3. Proyecto de plano del cementerio de Fernán Núñez, Pedro Nolasco Meléndez, 1860, AHN-SN, Toledo. 


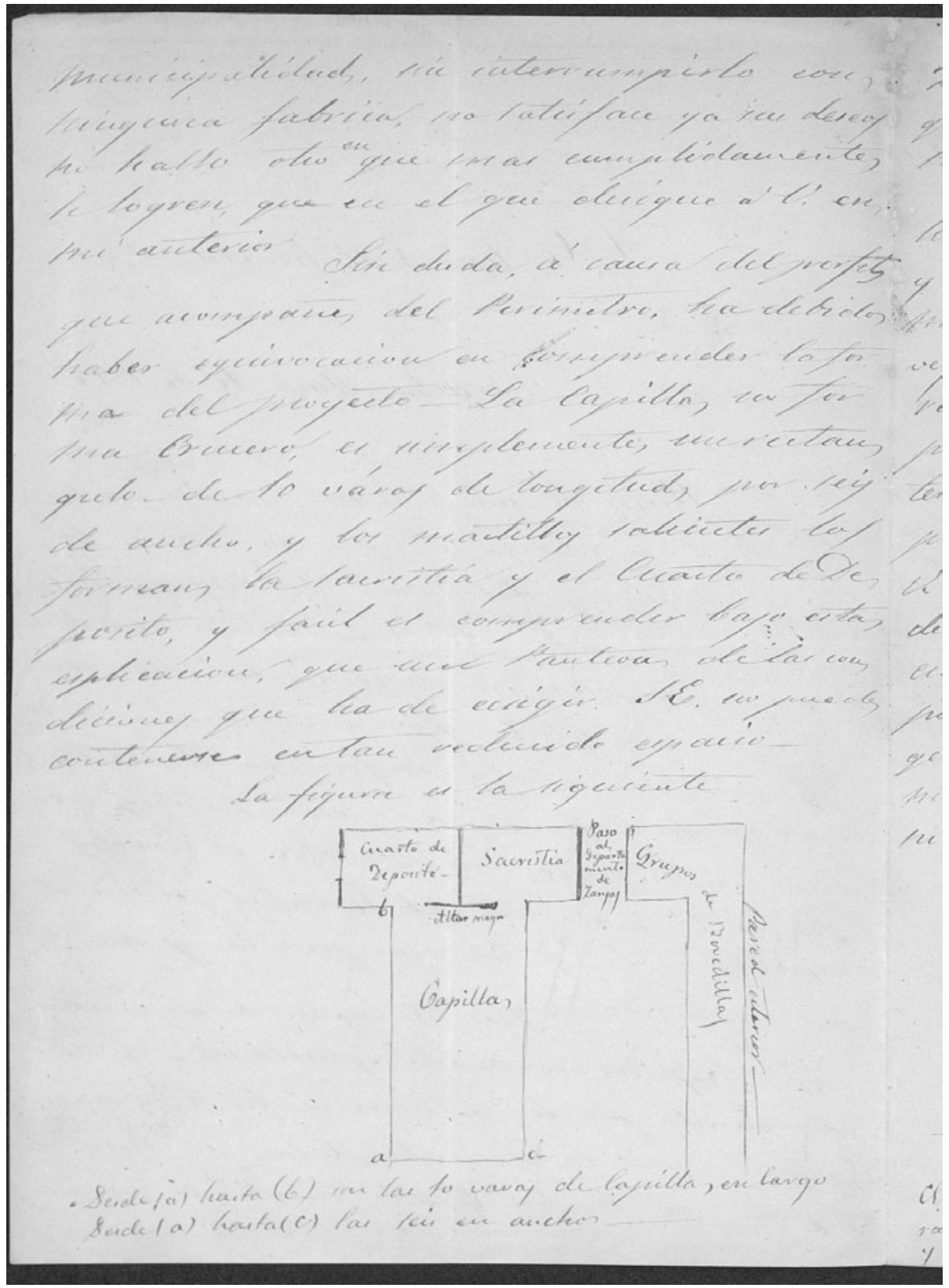

Figura 4. Croquis de la estructura, fachada principal y panteones del cementerio de Fernán Núñez, Pedro Nolasco Meléndez, 1860, AHN-SN, Toledo. 


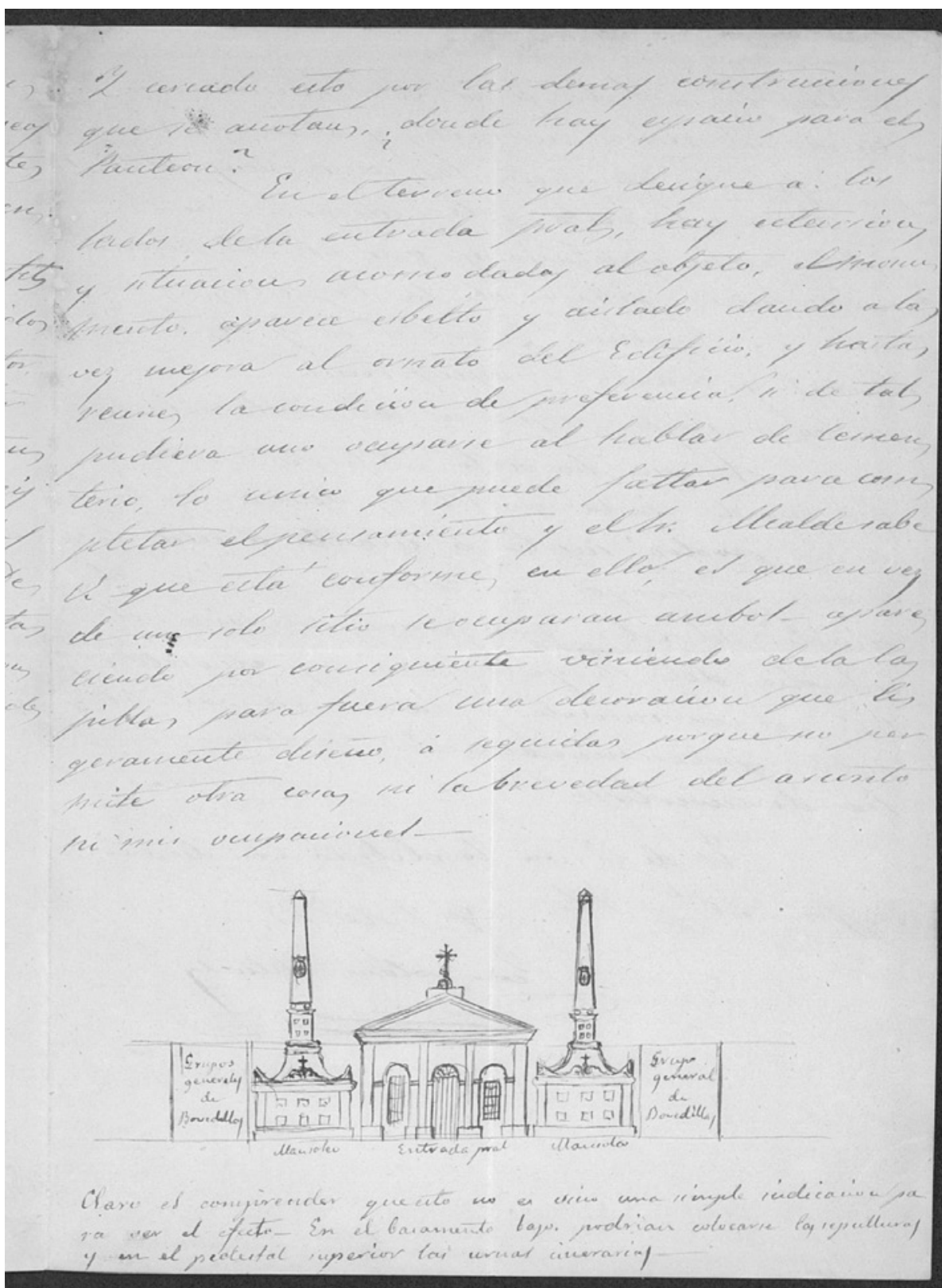

Figura 5. Croquis de la estructura, fachada principal y panteones del cementerio de Fernán Núñez, Pedro Nolasco Meléndez, 1860, AHN-SN, Toledo. 


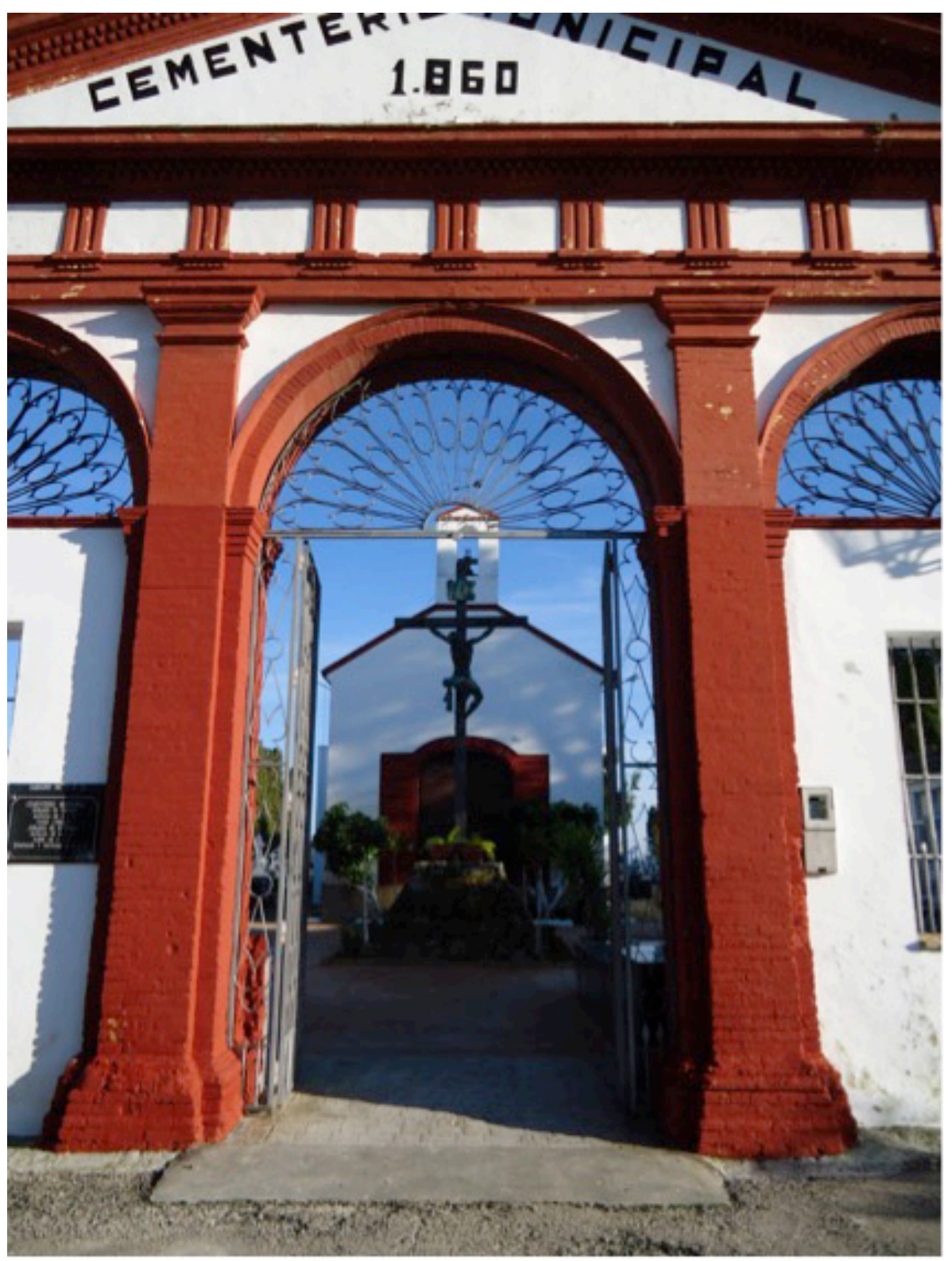

Figura 6. Fachada actual del cementerio de Fernán Núñez, 1860, Pedro Nolasco Meléndez, Fernán Núñez. 


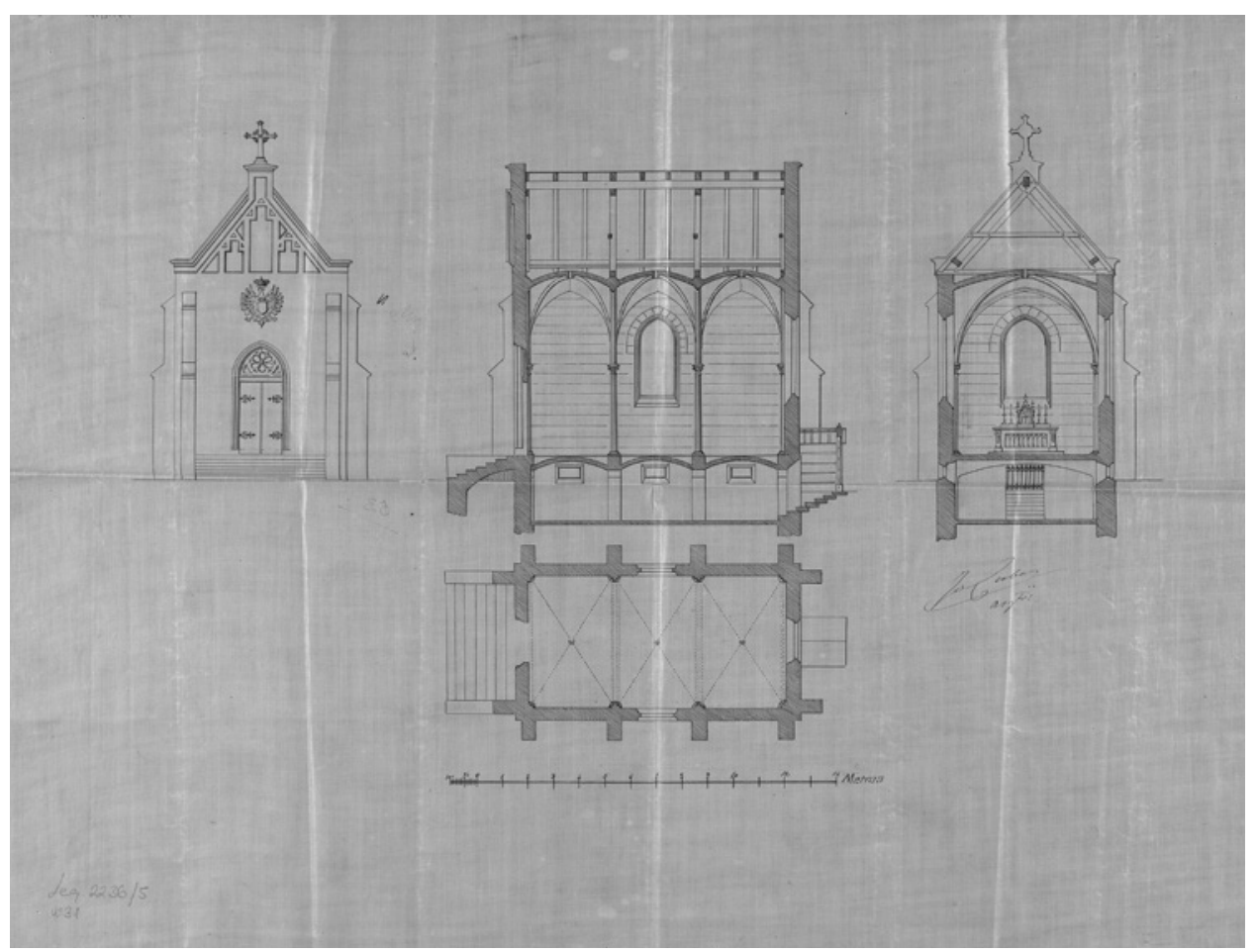

Figura 7. Plano del panteón de los III duques de Fernán Núñez en La Alameda en Madrid, 1879, Francisco de Cubas y González, AHN-SN, Toledo. 


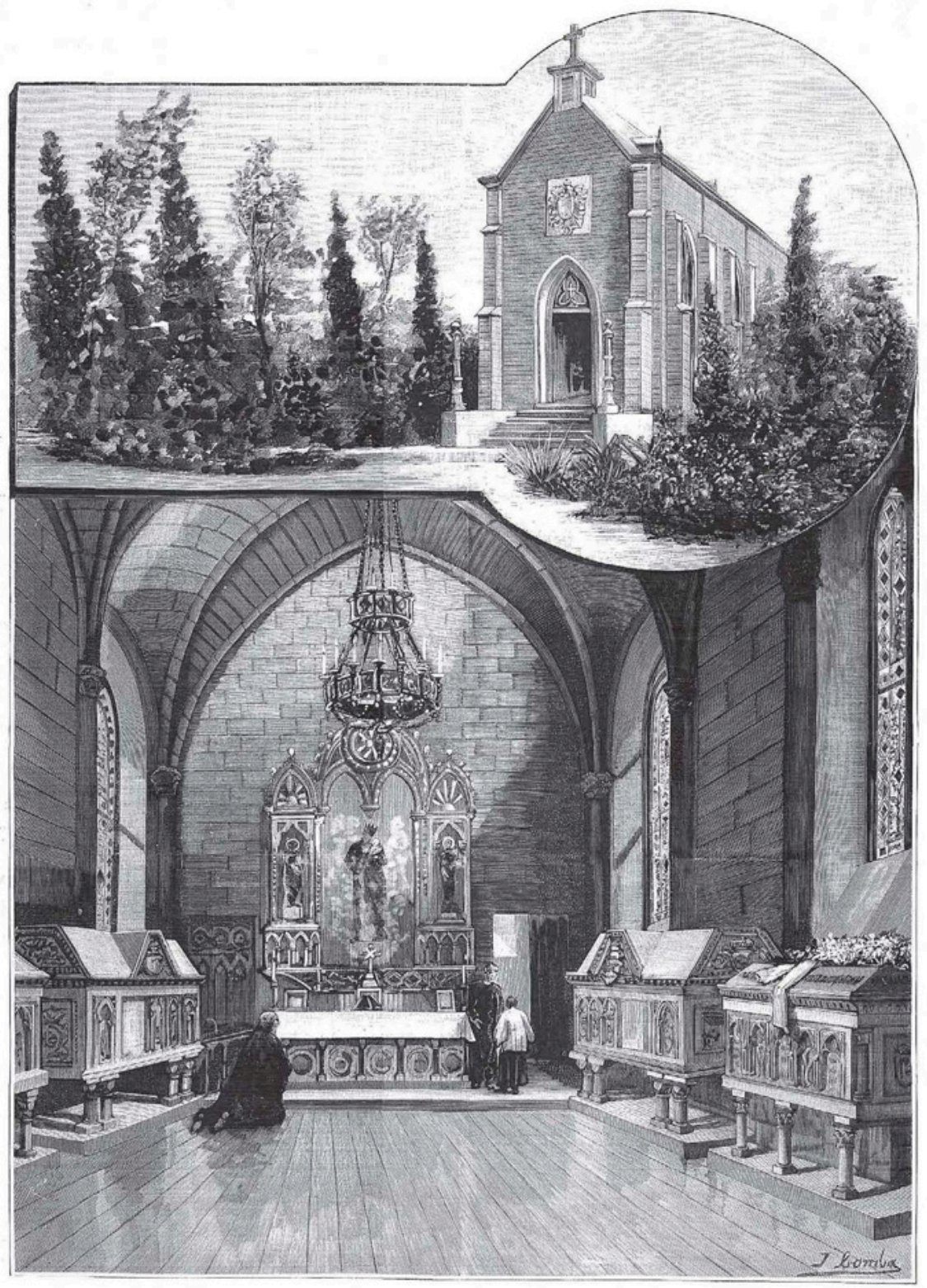

Figura 8. Exterior e interior del panteón de familia de los Excmos. Sres. duques de Fernán Núñez, Julio Comba, 1890, La Ilustración Española y Americana. 